\title{
Field Application of Chelated Calcium: Postharvest Effects on Cantaloupe and Honeydew Fruit Quality
}

\author{
Gene E. Lester ${ }^{1}$ and \\ Michael A. Grusak ${ }^{2}$
}

Additional index words. Cucumis melo, consumer taste preference, soluble solids concentration

Summary. Commercially grown honeydew fruit (Cucumis melo Inodorus group) and netted cantaloupe fruit (C. melo Reticulatus group) in lowhumidity regions of the U.S. are typically field packed, eliminating the possibility for postharvest chelatedcalcium dip treatments to extend fruit shelf life. In this study, calcium treatments were applied to orange-flesh honeydew fruit commercially grown in 2001 and 2002 in Sacramento Valley, Calif. and orange-fleshed netted cantaloupe fruit commercially grown in 2002 in Imperial Valley, Calif., and Rio Grande Valley, Texas. Aminoacid-chelated calcium and mannitolcomplexed calcium compounds were applied to field-grown plants at the rate of $2.3 \mathrm{~L} \cdot \mathrm{ha}^{-1}$ (1 qt/acre) at 0 , 1,2 , or 4 total applications during growth of honeydew and cantaloupe fruit. Applications were A) at female flowering, B) within 15 days (cantaloupe) or 20 days (honeydew) after flowering, C) within 30 days (cantaloupe) or 40 days (honeydew) after

\footnotetext{
We are grateful to the following for their participation and technical support in this study: Half-Moon Fruit and Produce Co. (Yolo, Calif.), Sandstone Marketing Inc. (Brawley, Calif.), Starr Produce Co. (Rio Grande City, Texas), Pedro Velasquez (Imperial Valley, Calif.), Isaac Castaneda (Sacramento Valley, Calif.), Tim Hartz (Univ. of Calif.-Davis), and Robert Meyer and David Hanna (USDA-ARS, Weslaco, Texas). This research was funded in part by the California Melon Research Board no. 58-6204-1-005 and by the USDA-ARS under CRIS no. 6204-43000-010-00D to G.E.L. and CRIS no. 6250-21520-041-00D to M.A.G. Use of company or product names by the USDA does not imply approval or recommendation of the product to the exclusion of others that may be suitable.

${ }^{1}$ Research plant physiologist, USDA-ARS, Kika de la Garza Subtropical Agricultural Research Center, 2413 E. Hwy. 83, Bldg. 200, Weslaco, TX 78596; e-mail glester@weslaco.ars.usda.gov.

${ }^{2}$ Research plant physiologist, USDA-ARS, Children's Nutrition Research Center, Department of Pediatrics, Baylor College of Medicine, 1100 Bates Street, Houston, TX 77030; e-mail mgrusak@bcm.tmc.edu.
} 
female flowering, and/or D) within 3 to 5 days before abscission. One application equaled (A) or (D), two applications equaled $(A+B)$ or $(C+$ $D)$ and four applications equaled ( $A$ $+B+C+D)$. Evaluations of fully abscised fruit were exterior and interior firmness, marketability, calcium concentrations, interior soluble solids concentration (sugars), and consumer preference (taste) following harvest and up to 3 weeks commercial/retail storage. Cantaloupe fruit at both locations did not appear to benefit from preharvest plant applications of calcium when compared to fruit from plants treated with water. Honeydew fruit, however, did and the benefit was observed both years. Honeydew fruit that received four preharvest plant applications of calcium, regardless of source, were generally superior in firmness, marketability, and had a higher calcium concentration than fruit from plants receiving water or one or two applications of calcium. Fruit sugars and taste were not affected by preharvest plant applications of calcium.

$\mathrm{P}$ ostharvest dipping fully ripened honeydew melons in organicchelated calcium versus EDTAchelated calcium or calcium chloride $\left(\mathrm{CaCl}_{2}\right)$ solutions has been shown to maintain stored honeydew fruit calcium concentration at levels found in recently abscised fruit, thus maintaining fruit tissue firmness, plasma membrane integrity, and extending storage life (Lester and Grusak, 1999, 2001). However, applying calcium to commercially grown melons as a postharvest dip is not suitable for melon growers in the desert southwestern U.S., or other low-humidity melon growing regions of the U.S. These growers box melons for shipment in the field at harvest, making a postharvest dip impractical. Although applications of calcium to melon fruit tissue disks (Lester, 1996), greenhouse-grown melons (Lester and Grusak, 1999), or commercially harvested melons (Lester and Grusak, 2001) has been reported, no studies have been reported for preharvest plant application of organicchelated calcium sources to commercial fields of either honeydew or netted cantaloupe during fruit production. Therefore, the objective of this study was to assess the benefits of preharvest plant applied applications of two commercial organic-chelated (amino-acid or mannitol) calcium formulations at different times during fruit growth and maturation. Fruit were evaluated for firmness, marketability, calcium concentrations, interior soluble solids concentration (sugars) and consumer preference (taste) following harvest and commercial storage for up to $22 \mathrm{~d}$.

\section{Materials and methods}

Calcium treatments. Either amino-acid-chelated calcium [calcium metalosate, 6\% actual calcium (Albion Laboratories, Inc., Clearfield, Utah)], or mannitol-complexed calcium [Folical, 10\% calcium (Monterey Chemical Co. Fresno, Calif.)] were applied to plants at the manufacturers recommended rate of $2.3 \mathrm{~L} \cdot \mathrm{ha}^{-1}$ in 75.7 to $113.6 \mathrm{~L}$ (20 to $30 \mathrm{gal})$ of water, sprayed at $345.0 \mathrm{kPa}\left(50 \mathrm{lb} / \mathrm{inch}^{2}\right)$ in California, or $621.0 \mathrm{kPa}\left(90 \mathrm{lb} / \mathrm{inch}^{2}\right)$ in Texas. Plants were sprayed by a commercial pesticide applicator $0,1,2$, or 4 total times during fruit growth to spring cantaloupes and to summer honeydew melons. Applications were A) at female flowering, B) within $15 \mathrm{~d}$ (cantaloupe) or $20 \mathrm{~d}$ (honeydew) after flowering, C) within $30 \mathrm{~d}$ (cantaloupe) or $40 \mathrm{~d}$ (honeydew) after female flowering, and/or D) within 3 to $5 \mathrm{~d}$ before abscission. Therefore, plants receiving one application $=[\mathrm{A}$ (one early) $]$ or $[\mathrm{D}$ (one late $)]$, two applications $=[\mathrm{A}$ $+\mathrm{B}$ (two early) $]$ or [C $+\mathrm{D}$ (two late) $]$ and four applications $=[\mathrm{A}+\mathrm{B}+\mathrm{C}+$ D (two early and two late)].

Plant material. Fully abscised fruit of 'Orange Dew' honeydew muskmelon grown in Sacramento Valley, Yolo County, Calif., 'Sol Real' netted cantaloupe muskmelon fruit grown in Imperial Valley, Imperial County, Calif., and 'Primo' grown in Rio Grande Valley, Starr County, Texas, free of defects, were harvested and shipped overnight to Weslaco, Texas. All fruit were washed in $0.53 \%$ (sodium hypochlorite) bleach, rinsed with tap water, then, within a treatment application randomized into lots for storage. Although field packing melons does not permit surface sterilizing fruit with a bleach solution, a bleach rinse was used to suppress postharvest disease decay in order to focus on physiological disorders associated with exogenous calcium applications. All fruit were placed in commercial honeydew or cantaloupe melon shipping boxes, then stored at $10 \pm 1{ }^{\circ} \mathrm{C}(50.0 \pm 1.8$ $\left.{ }^{\circ} \mathrm{F}\right)$ for honeydew or $4 \pm 1{ }^{\circ} \mathrm{C}(39.2 \pm$ $1.8^{\circ} \mathrm{F}$ ) for cantaloupe and $94 \% \pm 2 \%$ relative humidity ( $\mathrm{RH})$ for $4 \mathrm{~d}$ or 11 $\mathrm{d}$ for cantaloupe, and $11 \mathrm{~d}$ and $19 \mathrm{~d}$ for honeydew (simulating commercial shipping conditions) plus $3 \mathrm{~d}$ at $2 \mathrm{l} \pm \mathrm{l}$ ${ }^{\circ} \mathrm{C}\left(69.8 \pm 1.8^{\circ} \mathrm{F}\right)$ and $54 \% \pm 2 \% \mathrm{RH}$ (simulating retail conditions).

FRUIT FIRMNESS AND SOLUBLE SOLIDS CONCENTRATION DETERMINATIONS. Firmness of whole-fruit equatorial hypodermal-mesocarp tissue, minus the epidermis, and the middle-mesocarp tissue from an equatorially cut fruit, was measured using a V-tip probe [ 1 $\mathrm{cm}$ long $(0.4$ inch $) \times 5.08 \mathrm{~mm}$ diameter $(0.2$ inches $) \times 1 \mathrm{~mm}$ wide $(0.04$ inches)] attached to a force gauge (Mark-10, model MG20; Wagner Instruments, Greenwich, Conn.) and expressed as mean force in Newtons. Soluble solids concentration was determined on middle-mesocarp sections ( $1 \mathrm{~cm}$ long $\times 1 \mathrm{~cm}$ diameter) from three different locations along the fruit equatorial circumference (excluding the ground spot). Tissue sections were squeezed using a hand-held garlic press and soluble solids concentration on the expressed juice was determined using a temperature corrected, digital refractometer (Reichert Scientific Instruments, Buffalo, N.Y.).

Calcium determinations. Calcium concentrations were determined on hypodermal-mesocarp tissue taken from the equatorial region of the fruit and on middle-mesocarp tissue taken from three different locations around the fruit equatorial circumference (excluding the ground spot). The epidermis was removed followed by the hypodermal-mesocarp tissue with a ceramic vegetable peeler. Tissues were weighed immediately to determine fresh weight, and then dried at $70{ }^{\circ} \mathrm{C}\left(158.0^{\circ} \mathrm{F}\right)$ for $48 \mathrm{~h}$. Total calcium concentration per gram hypodermal-mesocarp or middle-mesocarp tissue fresh weight was determined, using atomic absorption spectroscopy, by a commercial plant tissue analysis lab.

Fruit marketability. Following storage, all fruit were rated for marketability (Lester and Shellie, 2002; Shellie and Lester, 2002); $0=0 \%$ of each fruit with disorders, $1=\leq 15 \%$ of each fruit with disorders, $2=\leq 50 \%$ of each fruit with disorders, $3=\leq 85 \%$ of each fruit with disorders, and $4=\leq 100 \%$ of each fruit with disorders. The data were arcsine transformed before analysis of variance (Little and Hills, 1978) and presented as percent marketable fruit. 
Data for marketability are presented as the percentage of fruit affected within a treatment.

Consumer preference determiNATIONS. Eating quality for fruit from each of the treatments by storage combinations was determined at individual stations by an untrained 25member preference panel. Each station displayed $2-\mathrm{cm}^{3}\left(0.12\right.$-inch $\left.{ }^{3}\right)$ melon cubes of middle-mesocarp tissue taken from the equatorial region of the fruit (excluding the ground spot). The panelists were randomly selected from the local USDA-ARS research community. A 9-cm (3.5-inch) line scale was used to rate overall fruit preference as previously described (Lester and Shellie, 1992).

Statistics. Analysis of variance was used to evaluate calcium treat- ments, firmness, marketability, and consumer preferences for the various data sets (SAS Inst., Cary, N.C.). Duncan's multiple range test $(P \leq 0.05)$ was used to discern between calcium application classifications when $F$ values were significant for main effects. Data are the average of eight, single-fruit replications per application per storage period.

Table 1. Firmness of 'Orange Dew' orange-flesh honeydew melon exterior with peel removed (exterior) and edible-flesh (interior) at harvest and following commercial storage. Amino-acid-chelated calcium [AA-Ca (calcium metalosate)] or mannitol-complexed calcium [MC-Ca (Foli-cal)] applied during fruit development to summer-grown plants in Sacramento Valley, Calif. Fruit were harvested at abscission in September 2001 and 2002.

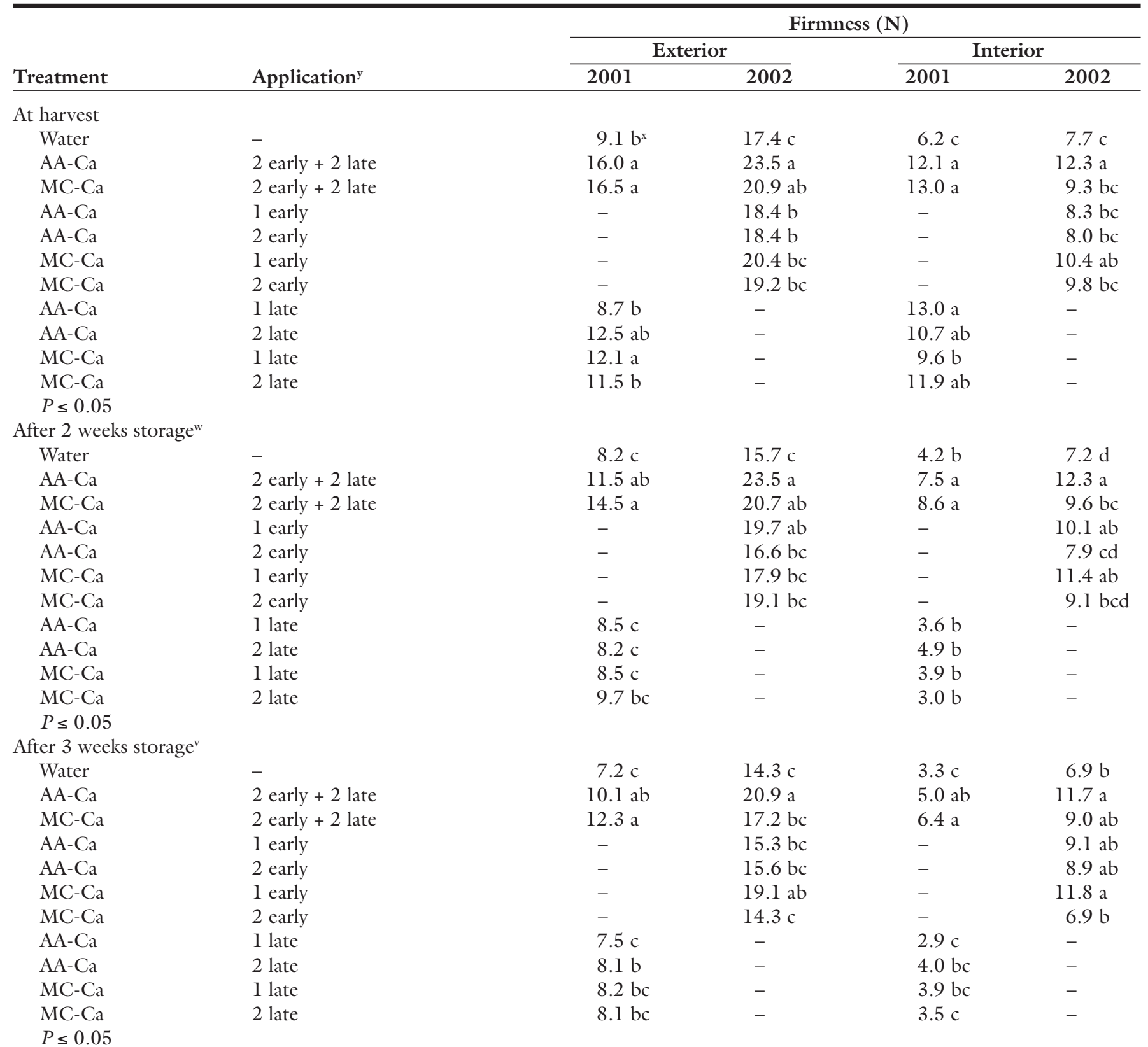

${ }^{\mathrm{z}} 1.0 \mathrm{~N}=0.225 \mathrm{lb}$ force.

ypplications: 1 early $=$ at female flowering, 2 early $=1$ early $+20 \mathrm{~d}$ later, 1 late $=3$ to $5 \mathrm{~d}$ before full-slip, 2 late $=40 \mathrm{~d}$ after female flowering +1 late. $(-)$ No treatment application conducted that year.

${ }^{x}$ Mean separation by Duncan's multiple range test. Any two means within a column and within a storage period followed by the same letter are not significantly different. Values are means $(\mathrm{n}=8)$.

${ }^{\mathrm{w}} 11 \mathrm{~d}$ at $10^{\circ} \mathrm{C}\left(50.0^{\circ} \mathrm{F}\right)$ and $95 \% \pm 3 \%$ relative humidity $(\mathrm{RH})$, plus $3 \mathrm{~d}$ at $21^{\circ} \mathrm{C}\left(69.8^{\circ} \mathrm{F}\right)$ and $50 \% \pm 3 \% \mathrm{RH}$.

$21 \mathrm{~d}$ at $10{ }^{\circ} \mathrm{C}$ and $95 \% \pm 3 \% \mathrm{RH}$, plus 3 d at $21{ }^{\circ} \mathrm{C}$ and $50 \% \pm 3 \% \mathrm{RH}$. 
Table 2. Calcium concentration of 'Orange Dew' orange-flesh honeydew melon exterior with peel removed and edible-flesh (interior) at harvest and following commercial storage. Amino-acid-chelated calcium [AA-Ca (calcium metalosate)] or mannitol-complexed calcium [MC-Ca (Foli-cal)] applied during fruit development to summer-grown plants in the Sacramento Valley, Calif. Fruit were harvested at abscission in September 2001 and 2002.

\begin{tabular}{|c|c|c|c|c|c|}
\hline \multirow[b]{3}{*}{ Treatment } & \multirow[b]{3}{*}{ Application $^{z}$} & \multicolumn{4}{|c|}{ Calcium $\left[\mu \mathrm{g} \cdot \mathrm{g}^{-1}(\mathrm{ppm})\right]$} \\
\hline & & \multicolumn{2}{|c|}{ Exterior } & \multicolumn{2}{|c|}{ Interior } \\
\hline & & 2001 & 2002 & 2001 & 2002 \\
\hline \multicolumn{6}{|l|}{ At harvest } \\
\hline Water & - & $2974 b^{y}$ & $3756 \mathrm{c}$ & $505 \mathrm{~b}$ & $417 \mathrm{~d}$ \\
\hline $\mathrm{AA}-\mathrm{Ca}$ & 2 early +2 late & 3748 a & $4872 \mathrm{a}$ & $654 \mathrm{a}$ & $667 a$ \\
\hline $\mathrm{MC}-\mathrm{Ca}$ & 2 early +2 late & 3748 a & $4443 \mathrm{~b}$ & $638 \mathrm{a}$ & $578 \mathrm{ab}$ \\
\hline $\mathrm{AA}-\mathrm{Ca}$ & l early & - & $4431 \mathrm{~b}$ & - & $470 \mathrm{~d}$ \\
\hline $\mathrm{AA}-\mathrm{Ca}$ & 2 early & - & $4374 \mathrm{~b}$ & - & $507 \mathrm{c}$ \\
\hline $\mathrm{MC}-\mathrm{Ca}$ & 1 early & - & $4482 \mathrm{~b}$ & - & $572 \mathrm{abc}$ \\
\hline MC-Ca & 2 early & - & $4387 \mathrm{~b}$ & - & $473 \mathrm{~cd}$ \\
\hline $\mathrm{AA}-\mathrm{Ca}$ & 1 late & $3377 \mathrm{ab}$ & - & $513 \mathrm{~b}$ & - \\
\hline $\mathrm{AA}-\mathrm{Ca}$ & 2 late & $3373 \mathrm{ab}$ & - & $634 \mathrm{ab}$ & - \\
\hline $\mathrm{MC}-\mathrm{Ca}$ & 1 late & 3298 a & - & $521 \mathrm{~b}$ & - \\
\hline $\mathrm{MC}-\mathrm{Ca}$ & 2 late & $2721 \mathrm{~b}$ & - & $618 \mathrm{ab}$ & - \\
\hline \multicolumn{6}{|c|}{$P \leq 0.05$} \\
\hline \multicolumn{6}{|c|}{ After 2 weeks storage } \\
\hline Water & - & $2677 \mathrm{bc}$ & $3175 \mathrm{c}$ & $502 \mathrm{~b}$ & $548 \mathrm{~cd}$ \\
\hline $\mathrm{AA}-\mathrm{Ca}$ & 2 early +2 late & $3342 \mathrm{ab}$ & $4429 \mathrm{a}$ & $642 \mathrm{a}$ & $671 \mathrm{a}$ \\
\hline $\mathrm{MC}-\mathrm{Ca}$ & 2 early +2 late & $3565 \mathrm{a}$ & $4119 \mathrm{ab}$ & $620 \mathrm{ab}$ & $627 \mathrm{a}$ \\
\hline $\mathrm{AA}-\mathrm{Ca}$ & l early & - & $3858 \mathrm{~b}$ & - & $502 \mathrm{~d}$ \\
\hline $\mathrm{AA}-\mathrm{Ca}$ & 2 early & - & $3830 \mathrm{~b}$ & - & $537 \mathrm{~b}$ \\
\hline MC-Ca & 1 early & - & $4087 \mathrm{~b}$ & - & $601 \mathrm{ab}$ \\
\hline MC-Ca & 2 early & - & $3991 \mathrm{~b}$ & - & $560 \mathrm{bc}$ \\
\hline $\mathrm{AA}-\mathrm{Ca}$ & 1 late & 2262 c & - & $457 \mathrm{c}$ & - \\
\hline $\mathrm{AA}-\mathrm{Ca}$ & 2 late & 2672 bc & - & $620 \mathrm{ab}$ & - \\
\hline MC-Ca & 1 late & $3020 \mathrm{abc}$ & - & $480 \mathrm{c}$ & - \\
\hline MC-Ca & 2 late & $2357 \mathrm{c}$ & - & $480 \mathrm{c}$ & - \\
\hline \multicolumn{6}{|c|}{$P \leq 0.05$} \\
\hline \multicolumn{6}{|c|}{ After 3 weeks storage ${ }^{w}$} \\
\hline Water & - & $2114 c$ & $3078 \mathrm{c}$ & $344 \mathrm{c}$ & $447 \mathrm{~b}$ \\
\hline $\mathrm{AA}-\mathrm{Ca}$ & 2 early +2 late & $2974 \mathrm{ab}$ & $4233 \mathrm{a}$ & $530 \mathrm{ab}$ & $522 \mathrm{a}$ \\
\hline MC-Ca & 2 early +2 late & $3351 \mathrm{a}$ & $3960 \mathrm{a}$ & $574 \mathrm{a}$ & 505 a \\
\hline $\mathrm{AA}-\mathrm{Ca}$ & l early & - & $3380 \mathrm{~b}$ & - & $472 \mathrm{ab}$ \\
\hline $\mathrm{AA}-\mathrm{Ca}$ & 2 early & - & $3241 \mathrm{bc}$ & - & $409 \mathrm{~b}$ \\
\hline $\mathrm{MC}-\mathrm{Ca}$ & 1 early & - & $3351 \mathrm{bc}$ & - & $465 \mathrm{ab}$ \\
\hline MC-Ca & 2 early & - & $3405 \mathrm{~b}$ & - & $465 \mathrm{ab}$ \\
\hline $\mathrm{AA}-\mathrm{Ca}$ & 1 late & $1515 \mathrm{~d}$ & - & $355 c$ & - \\
\hline $\mathrm{AA}-\mathrm{Ca}$ & 2 late & $2138 c$ & - & $485 \mathrm{~b}$ & - \\
\hline MC-Ca & 1 late & $2748 \mathrm{~b}$ & - & $400 \mathrm{c}$ & - \\
\hline MC-Ca & 2 late & $2357 \mathrm{c}$ & - & $334 \mathrm{c}$ & - \\
\hline$P \leq 0.05$ & & & & & \\
\hline
\end{tabular}

${ }^{\mathrm{z}}$ Applications: 1 early $=$ at female flowering, 2 early $=1$ early plus $20 \mathrm{~d}$ later, 1 late $=3$ to $5 \mathrm{~d}$ before full-slip, 2 late $=40 \mathrm{~d}$ after female flowering plus 1 late. $(-)=$ no treat ment application conducted that year.

${ }^{y}$ Mean separation by Duncan's multiple range test. Any two means within a column and within a storage period, followed by the same letter are not significantly different. Values are means $(\mathrm{n}=8)$.

${ }^{\mathrm{x}} \mathrm{ll} \mathrm{d}$ at $10^{\circ} \mathrm{C}\left(50.0^{\circ} \mathrm{F}\right)$ and $95 \% \pm 3 \%$ relative humidity $(\mathrm{RH})$, plus $3 \mathrm{~d}$ at $21^{\circ} \mathrm{C}\left(69.8^{\circ} \mathrm{F}\right)$ and $50 \% \pm 3 \% \mathrm{RH}$.

w $21 \mathrm{~d}$ at $10{ }^{\circ} \mathrm{C}$ and $95 \% \pm 3 \% \mathrm{RH}$, plus $3 \mathrm{~d}$ at $21^{\circ} \mathrm{C}, 50 \% \pm 3 \% \mathrm{RH}$.

\section{Results}

\section{Honeydew melons}

FIRMNESS. In 2001 and 2002, four applications of amino-acid-chelated calcium (AA-Ca) or mannitolcomplexed calcium (MC-Ca), versus control applications of water, resulted in significantly firmer readings of the fruit exterior tissues under the peel at harvest(Table 1). Fruit interior (edible tissue) firmness readings in 2001 from four applications of AA-Ca or MC-Ca, and in $2002 \mathrm{AA}-\mathrm{Ca}$ only, versus water, resulted in significantly firmer edible tissue. One or two applications of either calcium compound applied at female flowering, and/or within 20 $\mathrm{d}$ after flowering (early applications) or within $40 \mathrm{~d}$ after female flowering, and/or within 3 to $5 \mathrm{~d}$ before abscis- sion (late applications), were not always significantly better than water in affecting exterior or interior fruit firmness. Following storage of melons for 2 or 3 weeks, exterior and interior firmness was generally significantly firmer with four applications, of either plant applied calcium compound, than those receiving one or two early or late calcium applications or water. 
Table 3. Marketability of 'Orange Dew' orange-flesh honeydew melon exterior and edible-flesh (interior) following commercial storage. Amino-acid-chelated calcium [AA-Ca (calcium metalosate)] or mannitol-complexed calcium [MC-Ca (Folical)] applied during fruit development to summer-grown plants in the Sacramento Valley, Calif. Fruit were harvested at abscission in September 2001 and 2002.

\begin{tabular}{|c|c|c|c|c|c|}
\hline \multirow[b]{3}{*}{ Treatments } & \multirow[b]{3}{*}{ Applications $^{\mathrm{z}}$} & \multicolumn{4}{|c|}{ Marketability (\%) } \\
\hline & & \multicolumn{2}{|c|}{ Exterior } & \multicolumn{2}{|c|}{ Interior } \\
\hline & & 2001 & 2002 & 2001 & 2002 \\
\hline Water & - & $22 c^{x}$ & $49 \mathrm{c}$ & $69 \mathrm{c}$ & $86 \mathrm{~b}$ \\
\hline $\mathrm{AA}-\mathrm{Ca}$ & 2 early +2 late & $93 \mathrm{a}$ & $77 \mathrm{~b}$ & $100 \mathrm{a}$ & $100 \mathrm{a}$ \\
\hline $\mathrm{MC}-\mathrm{Ca}$ & 2 early +2 late & $100 \mathrm{a}$ & $95 \mathrm{a}$ & $100 \mathrm{a}$ & $100 \mathrm{a}$ \\
\hline $\mathrm{MC}-\mathrm{Ca}$ & 1 early & - & $49 \mathrm{c}$ & - & $100 \mathrm{a}$ \\
\hline MC-Ca & 2 early & - & $32 \mathrm{~d}$ & - & $100 \mathrm{a}$ \\
\hline $\mathrm{AA}-\mathrm{Ca}$ & 1 late & $48 \mathrm{~b}$ & - & $69 c$ & - \\
\hline $\mathrm{AA}-\mathrm{Ca}$ & 2 late & $54 \mathrm{~b}$ & - & $96 \mathrm{~b}$ & - \\
\hline MC-Ca & 1 late & $43 \mathrm{~b}$ & - & $69 c$ & - \\
\hline \multicolumn{6}{|c|}{$P \leq 0.05$} \\
\hline $\mathrm{AA}-\mathrm{Ca}$ & 2 early +2 late & $80 a$ & $37 \mathrm{a}$ & $96 a$ & $100 \mathrm{a}$ \\
\hline $\mathrm{MC}-\mathrm{Ca}$ & 2 early +2 late & $78 \mathrm{a}$ & $36 \mathrm{a}$ & $100 \mathrm{a}$ & $82 \mathrm{ab}$ \\
\hline $\mathrm{AA}-\mathrm{ca}$ & 1 early & - & $27 \mathrm{a}$ & - & $100 \mathrm{a}$ \\
\hline $\mathrm{AA}-\mathrm{Ca}$ & 2 early & - & $0 \mathrm{~b}$ & - & $100 \mathrm{a}$ \\
\hline MC-Ca & 1 early & - & $0 \mathrm{~b}$ & - & $100 \mathrm{a}$ \\
\hline MC-Ca & 2 early & - & $0 \mathrm{~b}$ & - & $100 \mathrm{a}$ \\
\hline $\mathrm{AA}-\mathrm{Ca}$ & 1 late & $42 \mathrm{~b}$ & - & $63 c$ & - \\
\hline $\mathrm{AA}-\mathrm{Ca}$ & 2 late & $42 \mathrm{~b}$ & - & $96 a$ & - \\
\hline MC-Ca & 1 late & $47 \mathrm{~b}$ & - & $90 \mathrm{~b}$ & - \\
\hline MC-Ca & 2 late & $47 \mathrm{~b}$ & - & $93 \mathrm{ab}$ & - \\
\hline$P \leq 0.05$ & & & & & \\
\hline
\end{tabular}

${ }^{2}$ Applications: 1 early $=$ at female flowering, 2 early $=1$ early plus $20 \mathrm{~d}$ later, 1 late $=3$ to $5 \mathrm{~d}$ before full-slip, 2 late $=40 \mathrm{~d}$ after female flowering plus 1 late. $(-)=$ no treatment application conducted that year.

${ }^{y} 11 \mathrm{~d}$ at $10^{\circ} \mathrm{C}\left(50.0^{\circ} \mathrm{F}\right)$ and $95 \% \pm 3 \%$ relative humidity $(\mathrm{RH})$, plus $3 \mathrm{~d}$ at $21^{\circ} \mathrm{C}\left(69.8^{\circ} \mathrm{F}\right)$ and $50 \% \pm 3 \% \mathrm{RH}$.

'Mean separation by Duncan's multiple range test. Any two means within a column and within a storage period, followed by the same letter are not significantly different. Values are means $(\mathrm{n}=8)$

w $21 \mathrm{~d}$ at $10{ }^{\circ} \mathrm{C}$ and $95 \% \pm 3 \% \mathrm{RH}$, plus $3 \mathrm{~d}$ at $21^{\circ} \mathrm{C}$ and $50 \% \pm 3 \% \mathrm{RH}$.

Calcium. In 2001 and 2002 , four applications of $\mathrm{AA}-\mathrm{Ca}$ or $\mathrm{MC}-\mathrm{Ca}$, versus water, resulted in significantly higher calcium concentrations in fruit exterior and interior tissues at harvest (Table 2). One or two, early or late, applications of either calcium compound were not always significantly higher in calcium than water. In both years, fruit from four applications of calcium stored for 2 weeks had higher exterior and interior calcium concentrations than water. After 3 weeks, for both years, fruit from four applications of calcium were significantly higher in tissue (exterior and interior) calcium concentrations than those treated with water.

MARKETABILITY. Following 2 or 3 weeks of storage, exterior and interior percent marketability of fruit from plants receiving four applications of $\mathrm{AA}-\mathrm{Ca}$ or $\mathrm{MC}-\mathrm{Ca}$, versus water, for most comparisons, were significantly greater (Table 3). Also, one or two applications of calcium applied early or late compared to water, for most comparisons, were significantly greater in exterior and interior marketability.

SugarS AND TASTE. Percent soluble solids concentration, within each production year, for all fruit was similar, regardless of calcium source or number of applications (Table 4). Sugars were lower by about $2.8 \%$ in 2002 than in 2001.

With few exceptions, consumer preference for all fruit ranged from like to like extremely (Table 4). Consumers usually gave fruit from water-treated plants the best taste ratings, but fruit from the water treatment were generally not significantly better than fruit from plants receiving calcium treatments; only a few of the calcium treatments resulted in fruit with significantly lower ratings, relative to water-treated controls.

\section{Cantaloupe melons}

Firmness. At harvest, whether grown in California or Texas, or having received preharvest plant applied calcium treatments or water, exterior or interior firmness readings were not consistently significantly different (Table 5 ). Following 1 or 2 weeks storage, fruit exterior or interior tissues from plants receiving applications of calcium were usually not significantly firmer than those from the water treatment.

Calcium. Atharvest, whether grown in California or Texas, calcium concentration was usually numerically the highest in both exterior and interior fruit tissues from plants having received four applications of AA-Ca, but the concentrations were not significantly higher than in fruit from the water treatment (Table 6 ). Following 1 or 2 weeks storage, fruit exterior and interior tissues from plants having received four applications of AA- 
Table 4. Soluble solids concentration and consumer preference of 'Orange Dew', orange-flesh honeydew melon edible-flesh at harvest and following commercial storage. Amino-acid-chelated calcium [AA-Ca (calcium metalosate)] or mannitol-complexed calcium [MC-Ca (Foli-cal)] applied during fruit development to summer-grown plants to the Sacramento Valley, Calif. Fruit were harvested at abscission in September 2001 and 2002.

\begin{tabular}{|c|c|c|c|c|c|}
\hline \multirow[b]{2}{*}{ Treatments } & \multirow[b]{2}{*}{ Applications ${ }^{\mathrm{z}}$} & \multicolumn{2}{|c|}{$\begin{array}{l}\text { Soluble solids concn } \\
(\%)\end{array}$} & \multicolumn{2}{|c|}{$\begin{array}{c}\text { Consumer preference } \\
\text { (taste) }\end{array}$} \\
\hline & & 2001 & 2002 & 2001 & 2002 \\
\hline \multicolumn{6}{|l|}{ At harvest } \\
\hline Water & - & $11.5 \mathrm{a}^{\mathrm{x}}$ & 9.9 a & $6.3 \mathrm{a}$ & $7.8 \mathrm{a}$ \\
\hline $\mathrm{AA}-\mathrm{Ca}$ & 2 early +2 late & $12.2 \mathrm{a}$ & $8.5 \mathrm{ab}$ & $6.6 \mathrm{a}$ & $6.9 \mathrm{ab}$ \\
\hline MC-Ca & 2 early +2 late & $11.9 \mathrm{a}$ & $8.5 \mathrm{ab}$ & $6.0 \mathrm{a}$ & $6.4 \mathrm{ab}$ \\
\hline $\mathrm{AA}-\mathrm{Ca}$ & 1 early & - & $9.6 \mathrm{ab}$ & - & $6.4 \mathrm{ab}$ \\
\hline $\mathrm{AA}-\mathrm{Ca}$ & 2 early & - & $8.4 \mathrm{ab}$ & - & $5.4 \mathrm{~b}$ \\
\hline MC-Ca & 1 early & - & $7.9 \mathrm{~b}$ & - & $6.1 \mathrm{ab}$ \\
\hline MC-Ca & 2 early & - & $9.6 \mathrm{ab}$ & - & $5.9 \mathrm{~b}$ \\
\hline $\mathrm{AA}-\mathrm{Ca}$ & 1 late & $10.5 \mathrm{a}$ & - & $5.7 \mathrm{a}$ & - \\
\hline $\mathrm{AA}-\mathrm{Ca}$ & 2 late & $11.0 \mathrm{a}$ & - & $3.9 \mathrm{~b}$ & - \\
\hline MC-Ca & 1 late & $10.6 \mathrm{a}$ & - & $6.2 \mathrm{a}$ & - \\
\hline $\mathrm{MC}-\mathrm{Ca}$ & 2 late & $11.3 \mathrm{a}$ & - & $5.9 \mathrm{a}$ & - \\
\hline \multicolumn{6}{|c|}{$P \leq 0.05$} \\
\hline \multicolumn{6}{|c|}{ After 2 weeks storagew } \\
\hline Water & - & $11.7 \mathrm{abc}$ & $8.5 \mathrm{a}$ & $7.0 \mathrm{a}$ & $7.0 \mathrm{a}$ \\
\hline $\mathrm{AA}-\mathrm{Ca}$ & 2 early \& 2 late & $12.0 \mathrm{a}$ & $7.7 \mathrm{a}$ & $5.4 \mathrm{ab}$ & $6.8 \mathrm{a}$ \\
\hline MC-Ca & 2 early \& 2 late & $11.8 \mathrm{ab}$ & $7.7 \mathrm{a}$ & $4.3 \mathrm{c}$ & $6.6 \mathrm{a}$ \\
\hline $\mathrm{AA}-\mathrm{Ca}$ & 1 early & - & $8.4 \mathrm{a}$ & - & $6.1 \mathrm{a}$ \\
\hline $\mathrm{AA}-\mathrm{Ca}$ & 2 early & - & $8.2 \mathrm{a}$ & - & $7.0 \mathrm{a}$ \\
\hline $\mathrm{MC}-\mathrm{Ca}$ & 1 early & - & $7.2 \mathrm{a}$ & - & $7.7 \mathrm{a}$ \\
\hline $\mathrm{MC}-\mathrm{Ca}$ & 2 early & - & $7.8 \mathrm{a}$ & - & $6.4 \mathrm{a}$ \\
\hline $\mathrm{AA}-\mathrm{Ca}$ & 1 late & $10.5 \mathrm{bc}$ & - & $5.3 \mathrm{ab}$ & - \\
\hline $\mathrm{AA}-\mathrm{Ca}$ & 2 late & $11.9 \mathrm{ab}$ & - & $4.7 \mathrm{~b}$ & - \\
\hline $\mathrm{MC}-\mathrm{Ca}$ & 1 late & $10.3 \mathrm{c}$ & - & $6.7 \mathrm{a}$ & - \\
\hline $\mathrm{MC}-\mathrm{Ca}$ & 2 late & $11.3 \mathrm{abc}$ & - & $6.1 \mathrm{ab}$ & - \\
\hline \multicolumn{6}{|c|}{$P \leq 0.05$} \\
\hline \multicolumn{6}{|c|}{ After 3 weeks storage ${ }^{v}$} \\
\hline Water & - & $10.3 \mathrm{~b}$ & $8.1 \mathrm{a}$ & $4.2 \mathrm{c}$ & $7.3 \mathrm{a}$ \\
\hline $\mathrm{AA}-\mathrm{Ca}$ & 2 early +2 late & $12.2 \mathrm{a}$ & $7.3 \mathrm{a}$ & $5.4 \mathrm{ab}$ & $7.4 \mathrm{a}$ \\
\hline $\mathrm{MC}-\mathrm{Ca}$ & 2 early +2 late & $13.1 \mathrm{a}$ & $8.3 \mathrm{a}$ & $5.6 \mathrm{ab}$ & $5.0 \mathrm{bc}$ \\
\hline $\mathrm{AA}-\mathrm{Ca}$ & 1 early & - & $8.3 \mathrm{a}$ & - & $6.8 \mathrm{a}$ \\
\hline $\mathrm{AA}-\mathrm{Ca}$ & 2 early & - & $7.9 \mathrm{a}$ & - & $5.8 \mathrm{ab}$ \\
\hline MC-Ca & 1 early & - & $7.4 \mathrm{a}$ & - & $3.7 \mathrm{c}$ \\
\hline $\mathrm{MC}-\mathrm{Ca}$ & 2 early & - & $7.8 \mathrm{a}$ & - & $6.1 \mathrm{ab}$ \\
\hline $\mathrm{AA}-\mathrm{Ca}$ & 1 late & $12.5 \mathrm{a}$ & - & $4.5 \mathrm{c}$ & - \\
\hline $\mathrm{AA}-\mathrm{Ca}$ & 2 late & $11.5 \mathrm{ab}$ & - & $5.6 \mathrm{ab}$ & - \\
\hline MC-Ca & 1 late & $10.8 \mathrm{~b}$ & - & $5.7 \mathrm{a}$ & - \\
\hline $\mathrm{MC}-\mathrm{Ca}$ & 2 late & $13.1 \mathrm{a}$ & - & $5.2 \mathrm{~b}$ & - \\
\hline$P<0.05$ & & & & & \\
\hline
\end{tabular}

${ }^{\mathrm{z}}$ Applications: 1 early $=$ at female flowering, 2 early $=1$ early plus $20 \mathrm{~d}$ later, 1 late $=3$ to $5 \mathrm{~d}$ before full-slip, 2 late $=40 \mathrm{~d}$ after female flowering plus 1 late. $(-)=$ no treat ment application conducted that year.

yPreference rating based on a scale means of 1 to 10 when 1 to $4=$ dislike extremely, 4.1 to $7=$ like, 7.1 to $10=$ like extremely. Values are $(\mathrm{n}=25)$.

${ }^{x}$ Mean separation by Duncan's multiple range test. Any two means within a column and within a storage period, followed by the same letter are not significantly different. Values are means $(\mathrm{n}=8)$.

${ }^{\mathrm{w}} 11 \mathrm{~d}$ at $10^{\circ} \mathrm{C}\left(50.0^{\circ} \mathrm{F}\right)$ and $95 \% \pm 3 \%$ relative humidity $(\mathrm{RH})$, plus $3 \mathrm{~d}$ at $21^{\circ} \mathrm{C}\left(69.8^{\circ} \mathrm{F}\right)$ and $50 \% \pm 3 \% \mathrm{RH}$.

v $21 \mathrm{~d}$ at $10^{\circ} \mathrm{C}$ and $95 \% \pm 3 \% \mathrm{RH}$, plus $3 \mathrm{~d}$ at $21^{\circ} \mathrm{C}$ and $50 \% \pm 3 \% \mathrm{RH}$.

Ca remained numerically the highest in calcium, and were generally significantly higher than water. However, all other calcium treatments were usually not significantly higher than water.

Marketability. Following I week of storage, exterior and interior percent marketability of fruit from plants receiving calcium versus water were not significantly different (Table 7). Fol- lowing 2 weeks storage, Texas grown fruit exterior and interior marketability from plants receiving calcium applications were significantly better than water. But in California-grown fruit, calcium treatments versus water were not statistically different for either exterior or interior percent marketability.

SugARS AND TASTE. Percent soluble solids concentration, within each grow- ing location, for all fruit was similar. Between locations, the concentration of sugars was lower in Texas than California-grown fruit.

With few exceptions, consumer preference for all fruit, regardless of location, ranged from like to like extremely (Table 8). Statistically significant differences were observed between certain treatments, but across 
Table 5. Firmness of 'Sol Real' (Calif.) and 'Primo' (Texas) orange-fleshed netted cantaloupe, with peel removed (exterior) and edible-flesh (interior) at harvest and following commercial storage. Amino-acid-chelated calcium [AA-Ca (calcium metalosate)] or mannitol-complexed calcium [MC-Ca (Foli-cal)] applied during fruit development to spring-grown plants harvested in June 2002 in the Imperial Valley, Calif., and May 2002 in the Rio Grande Valley, Texas.

\begin{tabular}{|c|c|c|c|c|c|}
\hline \multirow[b]{3}{*}{ Treatment } & \multirow[b]{3}{*}{ Application $^{y}$} & \multicolumn{4}{|c|}{ Firmness $(\mathbf{N})^{\mathrm{z}}$} \\
\hline & & \multicolumn{2}{|c|}{ Exterior } & \multicolumn{2}{|c|}{ Interior } \\
\hline & & Calif. & Texas & Calif. & Texas \\
\hline \multicolumn{6}{|l|}{ At harvest } \\
\hline Water & - & $56.9 \mathrm{a}^{\mathrm{x}}$ & $35.2 \mathrm{ab}$ & $15.9 \mathrm{a}$ & $11.8 \mathrm{ab}$ \\
\hline $\mathrm{AA}-\mathrm{Ca}$ & 2 early +2 late & $49.9 \mathrm{ab}$ & $38.9 \mathrm{ab}$ & $14.0 \mathrm{a}$ & $12.3 \mathrm{ab}$ \\
\hline $\mathrm{MC}-\mathrm{Ca}$ & 2 early +2 late & $51.1 \mathrm{ab}$ & $42.1 \mathrm{a}$ & $16.4 \mathrm{a}$ & $13.4 \mathrm{a}$ \\
\hline $\mathrm{AA}-\mathrm{Ca}$ & 1 early & $56.0 \mathrm{a}$ & $30.9 \mathrm{~b}$ & $15.2 \mathrm{a}$ & $10.8 \mathrm{~b}$ \\
\hline $\mathrm{AA}-\mathrm{Ca}$ & 2 early & $58.2 \mathrm{a}$ & $36.5 \mathrm{ab}$ & $16.4 \mathrm{a}$ & $11.8 \mathrm{ab}$ \\
\hline $\mathrm{MC}-\mathrm{Ca}$ & 1 early & $51.9 \mathrm{ab}$ & $34.9 \mathrm{ab}$ & $16.3 \mathrm{a}$ & $11.4 \mathrm{abc}$ \\
\hline MC-Ca & 2 early & $55.9 \mathrm{a}$ & $33.7 \mathrm{ab}$ & $16.5 \mathrm{a}$ & $13.1 \mathrm{a}$ \\
\hline $\mathrm{AA}-\mathrm{Ca}$ & 1 late & $39.0 \mathrm{~b}$ & $34.4 \mathrm{ab}$ & $13.9 \mathrm{a}$ & $10.6 \mathrm{bc}$ \\
\hline $\mathrm{AA}-\mathrm{Ca}$ & 2 late & $41.6 \mathrm{ab}$ & $36.5 \mathrm{ab}$ & $16.2 \mathrm{a}$ & $11.5 \mathrm{abc}$ \\
\hline MC-Ca & 1 late & $38.6 \mathrm{~b}$ & $31.2 \mathrm{~b}$ & $14.0 \mathrm{a}$ & $10.7 \mathrm{bc}$ \\
\hline MC-Ca & 2 late & $38.6 \mathrm{~b}$ & $31.2 \mathrm{~b}$ & $15.7 \mathrm{a}$ & $9.7 \mathrm{c}$ \\
\hline \multicolumn{6}{|l|}{$P \leq 0.05$} \\
\hline \multicolumn{6}{|c|}{ After 1 week storage ${ }^{w}$} \\
\hline Water & - & $21.8 \mathrm{~b}$ & $26.8 \mathrm{abc}$ & $10.2 \mathrm{a}$ & $8.4 \mathrm{bc}$ \\
\hline $\mathrm{AA}-\mathrm{Ca}$ & 2 early +2 late & $28.1 \mathrm{a}$ & $27.7 \mathrm{ab}$ & $10.2 \mathrm{a}$ & $9.7 \mathrm{~b}$ \\
\hline $\mathrm{MC}-\mathrm{Ca}$ & 2 early +2 late & $25.6 \mathrm{ab}$ & $28.3 \mathrm{a}$ & $8.3 \mathrm{~b}$ & $11.5 \mathrm{a}$ \\
\hline $\mathrm{AA}-\mathrm{Ca}$ & 1 early & $20.5 \mathrm{bcd}$ & $23.0 \mathrm{~d}$ & $8.0 \mathrm{~b}$ & 8.7 bc \\
\hline $\mathrm{AA}-\mathrm{Ca}$ & 2 early & $26.5 \mathrm{ab}$ & $24.8 \mathrm{abcd}$ & $10.2 \mathrm{a}$ & $7.5 \mathrm{c}$ \\
\hline MC-Ca & 1 early & $14.9 \mathrm{~d}$ & $23.9 \mathrm{bcd}$ & $7.3 \mathrm{~b}$ & $9.7 \mathrm{~b}$ \\
\hline $\mathrm{MC}-\mathrm{Ca}$ & 2 early & $20.5 \mathrm{bc}$ & $25.8 \mathrm{abcd}$ & $8.3 \mathrm{~b}$ & $8.9 \mathrm{bc}$ \\
\hline $\mathrm{AA}-\mathrm{Ca}$ & 1 late & $18.5 \mathrm{~cd}$ & $24.7 \mathrm{bcd}$ & $8.4 \mathrm{~b}$ & $7.8 \mathrm{c}$ \\
\hline $\mathrm{AA}-\mathrm{Ca}$ & 2 late & $22.9 \mathrm{abc}$ & $23.6 \mathrm{~cd}$ & $9.0 \mathrm{ab}$ & $8.4 \mathrm{bc}$ \\
\hline MC-Ca & 1 late & $18.5 \mathrm{~cd}$ & $26.8 \mathrm{abc}$ & $7.6 \mathrm{~b}$ & $8.5 \mathrm{bc}$ \\
\hline $\mathrm{MC}-\mathrm{Ca}$ & 2 late & $23.6 \mathrm{bc}$ & $25.5 \mathrm{abcd}$ & $8.5 \mathrm{~b}$ & $9.0 \mathrm{bc}$ \\
\hline \multicolumn{6}{|c|}{$P \leq 0.05$} \\
\hline \multicolumn{6}{|c|}{ After 2 weeks storage ${ }^{v}$} \\
\hline Water & - & $18.6 \mathrm{~cd}$ & $22.4 \mathrm{~b}$ & $9.9 \mathrm{ab}$ & $7.9 \mathrm{~b}$ \\
\hline $\mathrm{AA}-\mathrm{Ca}$ & 2 early +2 late & $24.1 \mathrm{a}$ & $25.1 \mathrm{ab}$ & $10.1 \mathrm{a}$ & $9.0 \mathrm{ab}$ \\
\hline $\mathrm{MC}-\mathrm{Ca}$ & 2 early +2 late & $22.7 \mathrm{ab}$ & $27.9 \mathrm{a}$ & $7.1 \mathrm{c}$ & $11.0 \mathrm{a}$ \\
\hline $\mathrm{AA}-\mathrm{Ca}$ & 1 early & $17.4 \mathrm{~cd}$ & $23.4 \mathrm{~b}$ & $8.6 \mathrm{bc}$ & $8.1 \mathrm{~b}$ \\
\hline $\mathrm{AA}-\mathrm{Ca}$ & 2 early & $18.6 \mathrm{~cd}$ & $23.9 \mathrm{ab}$ & $8.5 \mathrm{bc}$ & $8.7 \mathrm{~b}$ \\
\hline MC-Ca & l early & 19.4 bc & $22.9 \mathrm{~b}$ & $8.9 \mathrm{abc}$ & $9.2 \mathrm{ab}$ \\
\hline $\mathrm{MC}-\mathrm{Ca}$ & 2 early & $21.2 \mathrm{abc}$ & $22.9 \mathrm{~b}$ & $8.1 \mathrm{c}$ & $8.8 \mathrm{~b}$ \\
\hline $\mathrm{AA}-\mathrm{Ca}$ & 1 late & $17.2 \mathrm{~cd}$ & $22.5 \mathrm{~b}$ & $7.7 \mathrm{c}$ & $7.5 \mathrm{~b}$ \\
\hline $\mathrm{AA}-\mathrm{Ca}$ & 2 late & $21.3 \mathrm{abc}$ & $23.1 \mathrm{~b}$ & $8.5 \mathrm{bc}$ & $7.7 \mathrm{~b}$ \\
\hline MC-Ca & 1 late & $15.8 \mathrm{~d}$ & $20.7 \mathrm{~b}$ & $7.2 \mathrm{c}$ & $8.5 \mathrm{~b}$ \\
\hline MC-Ca & 2 late & $20.7 \mathrm{abc}$ & $20.9 \mathrm{~b}$ & $7.4 \mathrm{c}$ & $8.7 \mathrm{~b}$ \\
\hline$P \leq 0.05$ & & & & & \\
\hline
\end{tabular}

$\mathrm{z} 1.0 \mathrm{~N}=0.225 \mathrm{lb}$ force.

y Applications: 1 early $=$ at female flowering, 2 early $=1$ early plus $15 \mathrm{~d}$ later, 1 late $=3$ to $5 \mathrm{~d}$ before full-slip, 2 late $=30 \mathrm{~d}$ after female flowering plus 1 late

${ }^{x}$ Mean separation by Duncan's multiple range test. Any two means within a column and within a storage period, followed by the same letter are not significantly different. Values are means $(\mathrm{n}=8)$.

w $4 \mathrm{~d}$ at $4{ }^{\circ} \mathrm{C}\left(39.2^{\circ} \mathrm{F}\right)$ and $95 \% \pm 3 \%$ relative humidity $(\mathrm{RH})$, plus $3 \mathrm{~d}$ at $21^{\circ} \mathrm{C}\left(69.8^{\circ} \mathrm{F}\right)$ and $50 \% \pm 3 \% \mathrm{RH}$.

${ }^{v} 11 \mathrm{~d}$ at $4{ }^{\circ} \mathrm{C}$ and $95 \% \pm 3 \% \mathrm{RH}$, plus $3 \mathrm{~d}$ at $21{ }^{\circ} \mathrm{C}$ and $50 \% \pm 3 \% \mathrm{RH}$.

locations or storage times, no treatments were consistently different. Consumers usually gave fruit from calcium treated plants the best taste rating, but it was never always significantly better than fruit from plants receiving water (Table 8).

\section{Discussion}

Preharvest application of chelated calcium, especially four applications of either chelate source, consistently resulted in elevated mesocarp calcium concentrations, elevated fruit firmness, and enhanced marketability in honeydew fruit (relative to water-treated controls), but not in cantaloupe fruit (Tables 1-3 and 5-7). For cantaloupe, four preharvest calcium-chelate applications significantly enhanced cal- cium concentration of fruit interior and exterior tissues, but this effect was found inconsistently across locations, and only among some of the 1 - or 2-week stored fruit (relative to water-treated controls; Table 6). No effect of preharvest calcium-chelate application, with either melon type, was found on soluble solids concentration or consumer preference (Tables 4,8). 
Table 6. Calcium concentration of 'Sol Real' (Calif.) and 'Primo' (Texas) orange-fleshed netted cantaloupe, with peel removed (exterior) and edible-flesh (interior) at harvest and following commercial storage. Amino-acid-chelated calcium [AA$\mathrm{Ca}$ (calcium metalosate)] or mannitol-complexed calcium [MC-Ca (Foli-cal)] applied during fruit development to springgrown plants harvested in June 2002 in the Imperial Valley, Calif., and May 2002 in the Rio Grande Valley, Texas.

\begin{tabular}{|c|c|c|c|c|c|}
\hline \multirow[b]{3}{*}{ Treatments } & \multirow[b]{3}{*}{ Applications ${ }^{\mathrm{z}}$} & \multicolumn{4}{|c|}{ Calcium $\left[\mu g \cdot g^{-1}(p p m)\right]$} \\
\hline & & \multicolumn{2}{|c|}{ Exterior } & \multicolumn{2}{|c|}{ Interior } \\
\hline & & Calif. & Texas & Calif. & Texas \\
\hline \multicolumn{6}{|l|}{ At harvest } \\
\hline Water & - & $7743 \mathrm{ab}^{\mathrm{y}}$ & 9076 abc & $628 \mathrm{abc}$ & $1501 \mathrm{abc}$ \\
\hline $\mathrm{AA}-\mathrm{Ca}$ & 2 early +2 late & 8172 a & 9846 a & $747 \mathrm{a}$ & $1750 \mathrm{a}$ \\
\hline MC-Ca & 2 early +2 late & $7109 \mathrm{bc}$ & $9706 \mathrm{ab}$ & $735 \mathrm{ab}$ & $1635 \mathrm{a}$ \\
\hline $\mathrm{AA}-\mathrm{Ca}$ & 1 early & $6475 \mathrm{~cd}$ & $9143 \mathrm{ab}$ & $559 \mathrm{c}$ & $1319 \mathrm{bcd}$ \\
\hline $\mathrm{AA}-\mathrm{Ca}$ & 2 early & $6912 \mathrm{bc}$ & $9636 \mathrm{ab}$ & $629 \mathrm{abc}$ & $1482 \mathrm{abc}$ \\
\hline MC-Ca & 1 early & $5808 \mathrm{~d}$ & $8663 \mathrm{ab}$ & $630 \mathrm{abc}$ & $1276 \mathrm{bcd}$ \\
\hline MC-Ca & 2 early & $6148 \mathrm{~cd}$ & $8908 \mathrm{abc}$ & $718 \mathrm{a}$ & $1291 \mathrm{bcd}$ \\
\hline $\mathrm{AA}-\mathrm{Ca}$ & 1 late & $6407 \mathrm{~cd}$ & $9287 \mathrm{abc}$ & $671 \mathrm{abc}$ & $1059 \mathrm{~d}$ \\
\hline $\mathrm{AA}-\mathrm{Ca}$ & 2 late & $6971 \mathrm{bc}$ & $9308 \mathrm{abc}$ & $679 \mathrm{ab}$ & $1130 \mathrm{~d}$ \\
\hline $\mathrm{MC}-\mathrm{Ca}$ & 1 late & $6871 \mathrm{bc}$ & 8267 bc & $665 \mathrm{abc}$ & $1232 \mathrm{~cd}$ \\
\hline $\mathrm{MC}-\mathrm{Ca}$ & 2 late & $6254 c$ & $8310 \mathrm{bc}$ & $725 \mathrm{ab}$ & $1299 \mathrm{bcd}$ \\
\hline \multicolumn{6}{|c|}{$P \leq 0.05$} \\
\hline \multicolumn{6}{|c|}{ After 1 week storage ${ }^{x}$} \\
\hline Water & - & $5724 c$ & $8888 \mathrm{ab}$ & $625 \mathrm{~b}$ & $1323 \mathrm{a}$ \\
\hline $\mathrm{AA}-\mathrm{Ca}$ & 2 early +2 late & $7573 \mathrm{a}$ & $9511 \mathrm{ab}$ & $882 \mathrm{a}$ & $1557 \mathrm{a}$ \\
\hline $\mathrm{MC}-\mathrm{Ca}$ & 2 early +2 late & 5949 bc & $9580 \mathrm{a}$ & $814 \mathrm{a}$ & $1424 \mathrm{a}$ \\
\hline $\mathrm{AA}-\mathrm{Ca}$ & 1 early & $5542 \mathrm{c}$ & $8719 \mathrm{ab}$ & $617 \mathrm{~b}$ & $1375 \mathrm{a}$ \\
\hline $\mathrm{AA}-\mathrm{Ca}$ & 2 early & $5623 c$ & $8748 \mathrm{ab}$ & $713 \mathrm{ab}$ & 1299 a \\
\hline $\mathrm{MC}-\mathrm{Ca}$ & 1 early & $5803 \mathrm{bc}$ & $8552 \mathrm{ab}$ & $752 \mathrm{ab}$ & 1209 a \\
\hline $\mathrm{MC}-\mathrm{Ca}$ & 2 early & $7508 \mathrm{a}$ & $8452 \mathrm{ab}$ & $847 \mathrm{a}$ & 1289 a \\
\hline $\mathrm{AA}-\mathrm{Ca}$ & 1 late & 6448 abc & $9206 \mathrm{ab}$ & $742 \mathrm{ab}$ & 1396 a \\
\hline $\mathrm{AA}-\mathrm{Ca}$ & 2 late & $7001 \mathrm{ab}$ & $8467 \mathrm{ab}$ & $716 \mathrm{ab}$ & $1207 \mathrm{a}$ \\
\hline MC-Ca & 1 late & $5703 \mathrm{c}$ & $8722 \mathrm{ab}$ & $770 \mathrm{ab}$ & $1323 \mathrm{a}$ \\
\hline $\mathrm{MC}-\mathrm{Ca}$ & 2 late & $5966 \mathrm{bc}$ & $8285 \mathrm{~b}$ & $614 \mathrm{~b}$ & $1262 \mathrm{a}$ \\
\hline \multicolumn{6}{|c|}{$P \leq 0.05$} \\
\hline \multicolumn{6}{|c|}{ After 2 weeks storagew } \\
\hline Water & - & $5284 \mathrm{c}$ & $6685 \mathrm{~b}$ & $689 \mathrm{a}$ & $1268 c$ \\
\hline $\mathrm{AA}-\mathrm{Ca}$ & 2 early +2 late & $6769 a$ & 8817 a & $764 a$ & $1775 \mathrm{a}$ \\
\hline MC-Ca & 2 early +2 late & $5099 \mathrm{c}$ & 8818 a & $655 \mathrm{a}$ & $1363 \mathrm{bc}$ \\
\hline $\mathrm{AA}-\mathrm{Ca}$ & 1 early & $5088 c$ & 8697 a & $664 \mathrm{a}$ & $1663 \mathrm{ab}$ \\
\hline $\mathrm{AA}-\mathrm{Ca}$ & 2 early & $5644 \mathrm{bc}$ & 8747 a & $667 \mathrm{a}$ & $1276 \mathrm{c}$ \\
\hline MC-Ca & 1 early & $4580 \mathrm{~cd}$ & $7714 \mathrm{ab}$ & $600 \mathrm{a}$ & $1622 \mathrm{ab}$ \\
\hline MC-Ca & 2 early & $4485 \mathrm{~cd}$ & $7913 \mathrm{ab}$ & $632 \mathrm{a}$ & $1373 \mathrm{bc}$ \\
\hline AA-Ca & 1 late & $4965 c$ & $7310 \mathrm{~b}$ & $641 \mathrm{a}$ & $1502 \mathrm{abc}$ \\
\hline AA-Ca & 2 late & $6415 \mathrm{ab}$ & $8553 \mathrm{a}$ & $631 a$ & 1407 bc \\
\hline MC-Ca & 1 late & $3717 \mathrm{~d}$ & $7693 \mathrm{ab}$ & $611 \mathrm{a}$ & $1400 \mathrm{bc}$ \\
\hline MC-Ca & 2 late & $4078 \mathrm{~d}$ & $6883 \mathrm{~b}$ & $634 \mathrm{a}$ & $1363 \mathrm{bc}$ \\
\hline$P \leq 0.05$ & & & & & \\
\hline
\end{tabular}

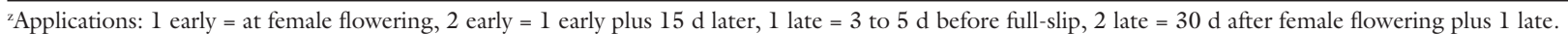

${ }^{y}$ Mean separation by Duncan's multiple range test. Any two means within a column and within a storage period, followed by the same letter are not significantly different. Values are means $(\mathrm{n}=8)$.

${ }^{\mathrm{x}} 4 \mathrm{~d}$ at $4{ }^{\circ} \mathrm{C}\left(39.2{ }^{\circ} \mathrm{F}\right)$ and $95 \% \pm 3 \%$ relative humidity $(\mathrm{RH})$, plus $3 \mathrm{~d}$ at $21^{\circ} \mathrm{C}\left(69.8{ }^{\circ} \mathrm{F}\right)$ and $50 \% \pm 3 \% \mathrm{RH}$.

${ }^{\mathrm{w}} \mathrm{ll} \mathrm{d}$ at $4{ }^{\circ} \mathrm{C}$ and $95 \% \pm 3 \% \mathrm{RH}$, plus $3 \mathrm{~d}$ at $21^{\circ} \mathrm{C}$ and $50 \% \pm 3 \% \mathrm{RH}$.

These results are consistent with those previously found following postharvest application of chelated calcium to fruit of honeydew or cantaloupe melons (Lester and Grusak, 1999). In that study, the calcium chelate permeated the netted epidermis of cantaloupe very poorly, and thus no increase was observed in the calcium concentration of the hypodermal mesocarp. Similarly, in the current study, it appears that the netted melons were unable to absorb calcium in sufficient amounts to have an influence on fruit quality traits (especially marketability). For the honeydew melons, on the other hand, it appears that with four treatments, enough calcium chelate was applied to the fruit, and enough of this calcium penetrated the non-netted epidermis to have an impact on the quality of the harvested fruit.
Interestingly, these results imply that the extra calcium which moved into honeydew fruit came from chelated calcium that was sprayed directly on the fruit, as is the case with preharvest calcium spraying of pear (Pyrus communis) trees (Rease and Drake, 1995). Although field spraying primarily covered the leaves with calcium chelate, some of the spray also would have reached the fruit, either as 
Table 7. Marketability of 'Sol Real'(Calif.) and 'Primo' (Texas) orange-fleshed netted cantaloupe, with peel removed (exterior) and edible-flesh (interior) at harvest and following commercial storage. Amino-acid-chelated calcium [AA-Ca (calcium metalosate)] or mannitol-complexed calcium [MC-Ca (Foli-cal)] applied during fruit development to spring-grown plants harvested in June 2002 in the Imperial Valley, Calif., and May 2002 in the Rio Grande Valley, Texas.

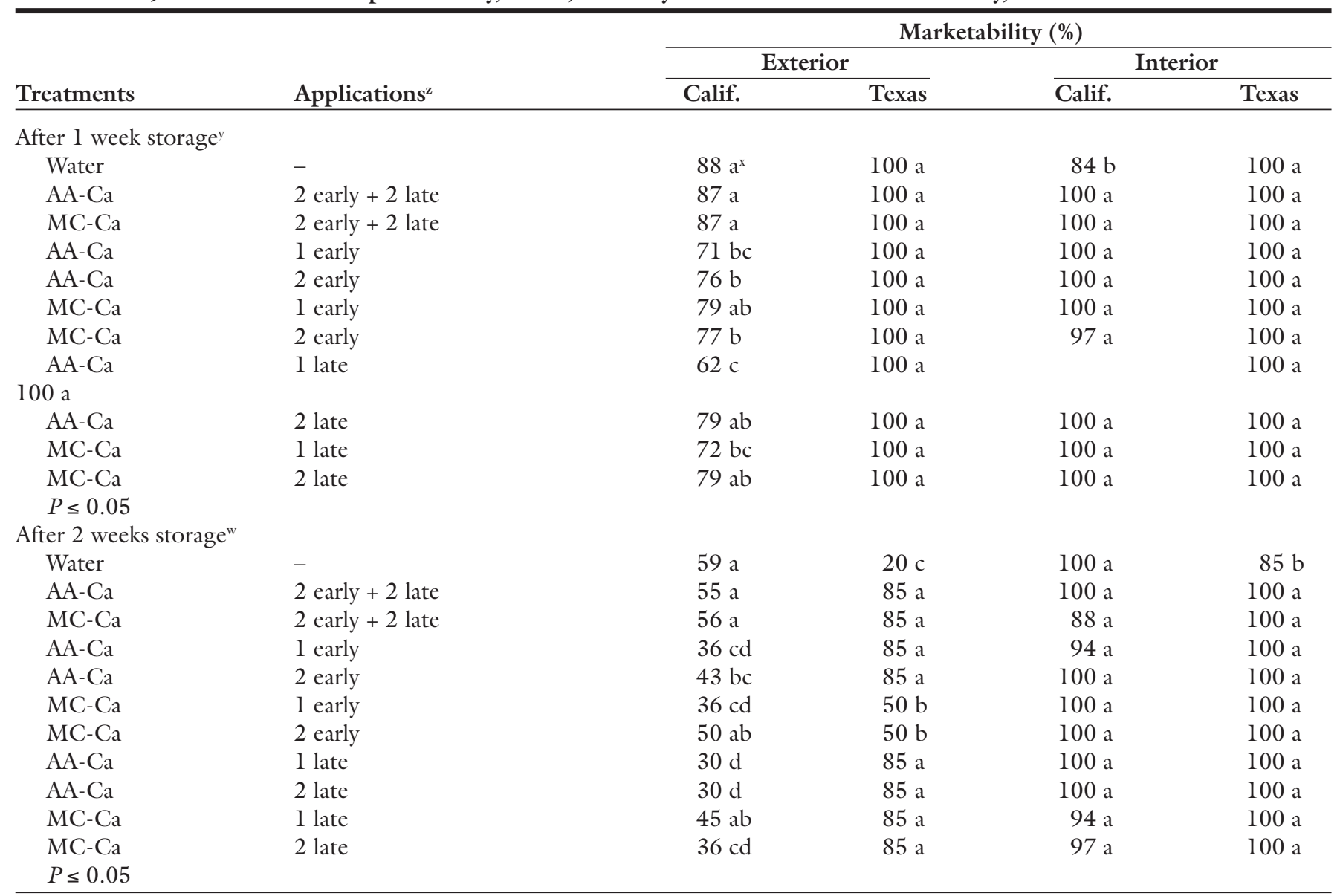

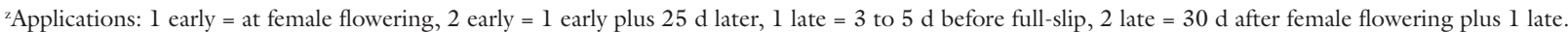
${ }^{y} 4 \mathrm{~d}$ at $4{ }^{\circ} \mathrm{C}\left(39.2{ }^{\circ} \mathrm{F}\right)$ and $95 \% \pm 3 \%$ relative humidity $(\mathrm{RH})$, plus $3 \mathrm{~d}$ at $21^{\circ} \mathrm{C}\left(69.8^{\circ} \mathrm{F}\right)$ and $50 \% \pm 3 \% \mathrm{RH}$.

${ }^{x}$ Mean separation by Duncan's multiple range test. Any two means within a column and within a storage period, followed by the same letter are not significantly different. Values are means $(\mathrm{n}=8)$.

${ }^{w} 11 \mathrm{~d}$ at $4{ }^{\circ} \mathrm{C}$ and $95 \% \pm 3 \% \mathrm{RH}$, plus $3 \mathrm{~d}$ at $21^{\circ} \mathrm{C}$ and $50 \% \pm 3 \% \mathrm{RH}$

foliar run-off, or as direct application to exposed melons on the ground. Secondary movement of calcium from leaves to fruit (i.e., potentially via the phloem transport pathway) appears not to have occurred, because both cantaloupe and honeydew melons should have responded positively to the treatments. This was not the case, and there is no reason to expect a difference in the way that leaves of the two melon types (which are both the same species) would have performed with respect to the foliar absorption of the calcium chelate, or to its subsequent partitioning. Furthermore, calcium is generally believed to move very poorly, if at all, in the phloem pathway (Clarkson, 1984). Thus, with direct application presumed to be the source of the supplemental calcium for honeydew melons, it is clear that plants should be sprayed in a way that will maximize coverage of the fruit themselves. Although four applications of chelated calcium proved the most beneficial to postharvest quality, further studies are needed to assess whether fewer sprayings with more concentrated calcium-chelate solution may also be effective.

The pertinent goal for melon growers is to have a highly marketable product of good nutritional quality. Although some improvements in the application techniques may yet arise following further investigations, our study has shown that preharvest calcium chelate application can improve postharvest fruit quality traits in honeydew melons, and that the use of this technology is a viable option for regions where melons are boxed in the field (or where postharvest applications of calcium chelate is impractical-in the packing shed-before boxing).

\section{Literature cited}

Clarkson, D.T. 1984. Calcium transport between tissues and its distribution in the plant. Plant Cell Environ. 7:449-456.

Lester, G. 1996. Calcium alters senescence rate of postharvest muskmelon fruit disks. Postharvest Biol. Technol. 7:91-96.

Lester, G. and K.C. Shellie. 1992. Postharvest sensory and physicochemical attributes of honeydew melon fruits. HortScience. 27: 1012-1014.

Lester, G and K.C. Shellie. 2002. Honeydew melon. In: K.C. Gross, C.Y. Wang, and M. Saltveit (eds.). 2002. The commercial storage of fruits, vegetables, and florist and nursery stocks. Draft copy for public review and comment only. U.S. Department of Agriculture, Agriculture Handbook 66. 8 Nov. 2002. <http://www.ba.ars.usda.gov/ hb66/073honeydew.pdfs.

Lester, G.E. and M.A. Grusak. 1999. Postharvest application of calcium and magnesium to honeydew and netted muskmelons: 
Table 8. Soluble solids concentration and consumer preference of 'Sol Real'(Calif.) and 'Primo' (Texas) orange-fleshed netted cantaloupe, with peel removed (exterior) and edible-flesh (interior) at harvest and following commercial storage. Amino-acid-chelated calcium [AA-Ca (calcium metalosate)] or mannitol-complexed calcium [MC-Ca (Foli-cal)] applied during fruit development to spring-grown plants harvested in June 2002 in the Imperial Valley, Calif., and May 2002 in the Rio Grande Valley, Texas.

Soluble solids concn

\begin{tabular}{cl} 
Treatment & Application $^{y}$ \\
\hline At harvest & \\
Water & - \\
AA-Ca & 2 early +2 late \\
MC-Ca & 2 early +2 late \\
AA-Ca & 1 early \\
AA-Ca & 2 early \\
MC-Ca & 1 early \\
MC-Ca & 2 early \\
AA-Ca & 1 late \\
AA-Ca & 2 late \\
MC-Ca & 1 late \\
MC-Ca & 2 late \\
P 00.05 &
\end{tabular}

After 1 week storagew

Water

AA-Ca

$\mathrm{MC}-\mathrm{Ca}$

AA-Ca

AA-Ca

$\mathrm{MC}-\mathrm{Ca}$

$\mathrm{MC}-\mathrm{Ca}$

AA-Ca

AA-Ca

$\mathrm{MC}-\mathrm{Ca}$

$\mathrm{MC}-\mathrm{Ca}$

$P \leq 0.05$

After 2 weeks storagev

$\begin{array}{ll}\text { Water } & - \\ \text { AA-Ca } & 2 \text { early }+2 \text { late } \\ \text { MC-Ca } & 2 \text { early }+2 \text { late } \\ \text { AA-Ca } & 1 \text { early } \\ \text { AA-Ca } & 2 \text { early } \\ \text { MC-Ca } & 1 \text { early } \\ \text { MC-Ca } & 2 \text { early } \\ \text { AA-Ca } & 1 \text { late } \\ \text { AA-Ca } & 2 \text { late } \\ \text { MC-Ca } & 1 \text { late } \\ \text { MC-Ca } & 2 \text { late } \\ P \leq 0.05 & \end{array}$

Calif.
$10.9 \mathrm{ab}^{\mathrm{x}}$
$10.6 \mathrm{ab}$
$10.8 \mathrm{ab}$
$12.0 \mathrm{a}$
$11.3 \mathrm{a}$
$11.1 \mathrm{a}$
$11.7 \mathrm{a}$
$10.5 \mathrm{ab}$
$11.6 \mathrm{a}$
$9.5 \mathrm{~b}$
$10.8 \mathrm{ab}$

$10.5 c$

$11.6 \mathrm{ab}$

$11.2 \mathrm{bc}$

$11.1 \mathrm{bc}$

$12.5 \mathrm{ab}$

$11.7 \mathrm{ab}$

$12.6 \mathrm{a}$

$11.6 \mathrm{ab}$

$12.4 \mathrm{ab}$

$11.9 \mathrm{ab}$

$11.2 \mathrm{bc}$
$10.1 \mathrm{~b}$
$11.1 \mathrm{a}$
$10.4 \mathrm{ab}$
$10.6 \mathrm{a}$
$11.3 \mathrm{a}$
$10.4 \mathrm{ab}$
10.9 a
$10.0 \mathrm{~b}$
$10.8 \mathrm{a}$
$11.4 \mathrm{a}$
$9.7 \mathrm{~b}$

Consumer preference ${ }^{\mathrm{z}}$

(\%)

Texas

$8.3 \mathrm{ab}$

$8.2 \mathrm{ab}$

$7.6 \mathrm{bc}$

$8.0 \mathrm{abc}$

$8.3 \mathrm{ab}$

$7.8 \mathrm{bc}$

$7.5 \mathrm{bc}$

$8.4 \mathrm{ab}$

$9.2 \mathrm{a}$

$6.6 \mathrm{c}$

$7.4 \mathrm{bc}$
$8.3 \mathrm{ab}$
$7.7 \mathrm{ab}$
$8.8 \mathrm{ab}$
$7.6 \mathrm{~b}$
$8.4 \mathrm{ab}$
$8.4 \mathrm{ab}$
$8.1 \mathrm{ab}$
$8.4 \mathrm{ab}$
$9.2 \mathrm{a}$
$7.9 \mathrm{ab}$
$8.9 \mathrm{ab}$

$8.0 \mathrm{ab}$
$7.9 \mathrm{ab}$
$11.0 \mathrm{a}$
$7.9 \mathrm{~b}$
$8.4 \mathrm{~b}$
$9.2 \mathrm{ab}$
$8.8 \mathrm{~b}$
$8.3 \mathrm{~b}$
$8.6 \mathrm{~b}$
$8.5 \mathrm{~b}$
$8.7 \mathrm{~b}$

$8.0 \mathrm{ab}$

$1.0 \mathrm{a}$

$7.9 \mathrm{~b}$

$8.4 \mathrm{~b}$

$9.2 \mathrm{ab}$

$8.8 \mathrm{~b}$

$8.3 \mathrm{~b}$

$8.5 \mathrm{~b}$

$8.7 \mathrm{~b}$

\begin{tabular}{ll}
\multicolumn{2}{c}{ (taste) } \\
\hline Calif. & Texas
\end{tabular}

${ }^{2}$ Preference rating based on a scale means of 1 to 10 when 1 to $4=$ dislike extremely, 4.1 to $7=$ like, 7.1 to $10=$ like extremely. Values are $(\mathrm{n}=25)$.

ypplications: 1 early = at female flowering, 2 early = 1 early plus $20 \mathrm{~d}$ later, 1 late $=3$ to $5 \mathrm{~d}$ before full-slip, 2 late $=40 \mathrm{~d}$ after female flowering plus 1 late

${ }^{x}$ Mean separation by Duncan's multiple range test. Any two means within a column and within a storage period, followed by the same letter are not significantly different. Values are $(\mathrm{n}=8)$.

${ }^{w} 4 \mathrm{~d}$ at $4{ }^{\circ} \mathrm{C}\left(39.2{ }^{\circ} \mathrm{F}\right)$ and $95 \% \pm 3 \%$ relative humidity $(\mathrm{RH})$, plus $3 \mathrm{~d}$ at $21^{\circ} \mathrm{C}\left(69.8^{\circ} \mathrm{F}\right)$ and $50 \% \pm 3 \% \mathrm{RH}$

${ }^{\mathrm{v}} 11 \mathrm{~d}$ at $4{ }^{\circ} \mathrm{C}$ and $95 \% \pm 3 \% \mathrm{RH}$, plus $3 \mathrm{~d}$ at $21^{\circ} \mathrm{C}$ and $50 \% \pm 3 \% \mathrm{RH}$.

effects on tissue ion concentrations, quality, and senescence. J. Amer. Soc. Hort. Sci. 124:545-552.

Lester, G.E. and M.A. Grusak. 2001. Postharvest application of chelated and nonchelated calcium dip treatments to commercially grown honeydew melons: Effects on peel attributes, tissue calcium concentration, quality and consumer preference following storage. HortTechnology 11:561-566.
Little, T.M. and F.J. Hills. 1978. Agricultural experimental design and analysis. Wiley. New York.

Rease, J.T. and S.R. Drake. 1995. Calcium sprays and timing affect fruit calcium concentrations, yield, fruit weight, and cork spot of 'Anjou' pears. HortScience 30:1037-1039.

Shellie, K.C. and G. Lester. 2002. Netted melons. In: K.C. Gross, C.Y. Wang, and
M. Saltveit (eds.). 2002. The commercial storage of fruits, vegetables, and florist and nursery stocks. Draft copy for public review and comment only. U.S. Department of Agriculture, Agriculture Handbook 66. 8 Nov. 2002. <http://www.ba.ars.usda.gov/ hb66/093nettedmelon.pdf>. 